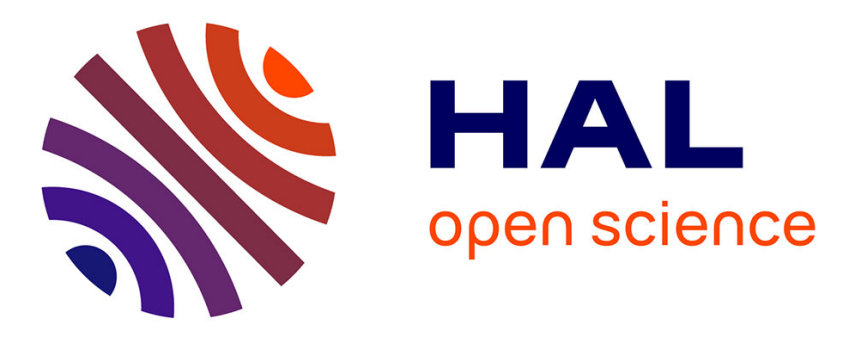

\title{
Optoelectronic cross-injection locking of a dual-wavelength photonic integrated circuit for low-phase-noise millimeter-wave generation
}

Gaël Kervella, Frederic van Dijk, Grégoire Pillet, Marco Lamponi, Mourad

Chtioui, Loïc Morvan, Mehdi Alouini

\section{To cite this version:}

Gaël Kervella, Frederic van Dijk, Grégoire Pillet, Marco Lamponi, Mourad Chtioui, et al.. Optoelectronic cross-injection locking of a dual-wavelength photonic integrated circuit for low-phase-noise millimeter-wave generation. Optics Letters, 2015, 40 (15), pp.3655. 10.1364/OL.40.003655 . hal02904707

\section{HAL Id: hal-02904707 \\ https://hal.science/hal-02904707}

Submitted on 22 Jul 2020

HAL is a multi-disciplinary open access archive for the deposit and dissemination of scientific research documents, whether they are published or not. The documents may come from teaching and research institutions in France or abroad, or from public or private research centers.
L'archive ouverte pluridisciplinaire HAL, est destinée au dépôt et à la diffusion de documents scientifiques de niveau recherche, publiés ou non, émanant des établissements d'enseignement et de recherche français ou étrangers, des laboratoires publics ou privés. 


\title{
Optoelectronic cross-injection locking of a dual- wavelength photonic integrated circuit for low phase noise millimeter-wave generation
}

\author{
Gaël Kervella, ${ }^{1}$ Frederic Van Dijk, ${ }^{1}$ Grégoire Pillet, ${ }^{2}$ Marco Lamponi,,${ }^{1, *}$ Mourad Chtioui, ${ }^{4}$ Loïc \\ Morvan², Mehdi Alouini, ${ }^{2,3}$ \\ ${ }^{1}$ III-V Lab, a joint Laboratory of "Alcatel Lucent Bell Labs", "Thales Research \& Technology" and "CEA-LETI", 1 avenue \\ Augustin Fresnel 91127 Palaiseau, France \\ ${ }^{2}$ Thales Research and Technology - France, 1 avenue Augustin Fresnel 91127 Palaiseau, France \\ ${ }^{3}$ Institut de Physique de Rennes, 263 Avenue Général Leclerc, 35700 Rennes \\ ${ }^{4}$ Thales Air Systems, 1, 91470, Limours, France \\ ${ }^{*}$ now with Huawei Technologies · Caliopa, Technologiepark 199052 Zwijnaarde, Belgium
}

\begin{abstract}
We report on the stabilization of a $90 \mathrm{GHz}$ millimeter-wave signal generated from a fully integrated photonic circuit. The chip consists of two DFB single mode lasers which optical signals are combined on a fast photodiode to generate a largely tunable heterodyne beat note. We generate an optical comb from each laser with a microwave synthesizer, and by self-injecting the resulting signal, we mutually correlate the phase noise of each DFB and we stabilize the beatnote on a multiple of the frequency delivered by the synthesizer. The performances achieved beat note linewidth below $30 \mathrm{~Hz}$.
\end{abstract}

Keywords : Lasers, distributed-feedback, Heterodyne, Integrated optics, Radio frequency photonics

Millimeter-wave (mmW) frequency range (between 30 $\mathrm{GHz}$ and $300 \mathrm{GHz}$ ) is of great interest for broadband wireless communications mainly because of the available bandwidth and the good directivity of the signal [1]. Optical solutions for generation and modulation of high frequency signals have two main advantages. First, the mmW carrier can be generated by heterodyning two optical tones. Second, the data can be modulated on the carrier by electro-optical modulation of the optical power.

The two optical tones can be issued by a dual wavelength laser that can be easily tuned. The optoelectronic conversion requires a high-speed photodetector. Monolithic integration in an indium phosphide chip of all these elements has already been demonstrated [2] and promises cost effective production, compact packaging, and fewer alignment issues.

In a previous paper, we presented the generation of $\mathrm{mmW}$ signal tunable from 5 to $110 \mathrm{GHz}$ with such an integrated source [3]. The main issue was related to the large beat note linewidth which can be detrimental to the transmission when the source is used in high data-rate wireless communications. Some solutions based on phaselocking techniques have been investigated. (i) The first solution consists in phase-locking the generated signal onto a reference oscillator (LO) [4] by applying a feedback signal to correct the beatnote frequency. The spectral purity of the LO is then transferred to the beatnote. This requires the optical linewidth of each of the two optical tones to be narrow enough and the feedback electronics to be fast enough, with a short time delay. (ii) The second solution consists in optical injection of an optical reference such as an optical frequency comb. This was successfully demonstrated on devices very similar to those presented here [5]. An alternative an elegant solution using an auxiliary third laser has been also demonstrated taking advantage of four wave mixing mechanism un the auxiliary laser [8]. (iii) The third solution avoids the use of an external optical source. It consists in mutually self optical injection-locking of the two DFB lasers after electrooptical modulation with a stable electronic oscillator. This was already demonstrated with a solid-state dualfrequency laser [6] and with a semiconductor dualwavelength laser [7].

In this paper we present the first cross injection locking of a $90 \mathrm{GHz} \mathrm{mmW}$ signal issued by a fully integrated and largely tunable $\mathrm{mmW}$ photonic source.

The photonic integrated circuit (PIC) under consideration is shown in Figure 1. It includes two optical distributed feedback lasers placed on each side of the chip (DFB1 and DFB2) working around $1555 \mathrm{~nm}$. Their beams are combined with a $2 \times 2$ multimode interference (MMI) coupler. Semi-conductor amplifiers (SOA) are placed at different positions of the circuit to amplify and adjust the signals levels. At last, the optical beat signal is sent onto a UTC photodiode integrated on the same chip where it is converted into an electrical $\mathrm{mmW}$ signal. If we call $\lambda_{1}$ and $\lambda_{2}$ (resp. $v_{1}$ and $v_{2}$ ) the wavelengths (resp. frequencies) of the two DFB lasers, the $\mathrm{mmW}$ frequency is:

$$
f=\frac{c\left(\lambda_{2}-\lambda_{1}\right)}{\lambda_{2} \lambda_{1}}=\nu_{2}-\nu_{1}
$$

An integrated electro-optical modulator is inserted before the UTC photodiode in order to modulate the beatnote which is used as a carrier frequency. The photodiode output can then be directly connected to a mmW antenna for free space data transmission. Note that an optical access to the DFB lasers is still possible on the other side of the chip (left-hand side in Fig. 1). This optical access enables monitoring of the optical signals as well as 
optical feedback as shown in the following. All these functions are realized in a single $4.4 \mathrm{mmx} 0.7 \mathrm{~mm}$ device.

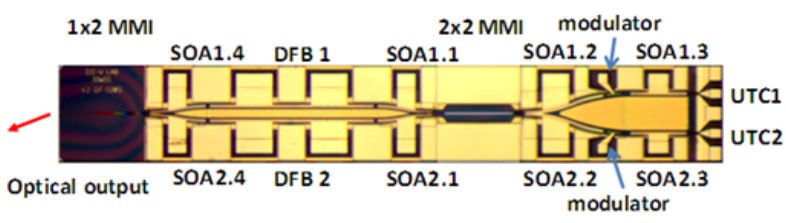

Fig.1. Microscope view of the integrated photonic chip.

We already demonstrated that our PIC allows a wide and continuous tuning of the beatnote frequency from 5 to $105 \mathrm{GHz}$ [9] thanks to the easy tuning of the DFBs wavelengths by adjusting the driving current. Moreover, as already shown in [10], the electro-optical modulator integrated in the chip enables high data rate transmissions: back-to-back transmission up to $4 \mathrm{Gbit} / \mathrm{s}$ was demonstrated. Nevertheless, we observed that the data rate is in practice limited to $200 \mathrm{Mbit} / \mathrm{s}$ for a wireless transmission. We then found that the data rate limitation was due to the high phase noise level of the $\mathrm{mmW}$ carrier. Indeed, in addition to the intrinsic optical phase noise associated to the natural linewidth of each of the DFB lasers, additive phase noise appears because of parasitic reflections in the photonic integrated circuit and on the back of the photodetectors that are modulated by the data stream applied to the electro-optical modulator.

Consequently, these effects have to be reduced and the $\mathrm{mmW}$ carrier phase noise improved in order to make this PIC fully compatible with wireless transmissions. The proposed solution involves optical injection locking (OIL). It is well known that injecting a slave laser (SL) with a low-phasenoise master laser (ML) forces the SL to operate at the same wavelength and to have a phase noise similar to the ML [11], provided that the wavelength of the two lasers are sufficiently close and that the injected optical power is optimized. If the $\mathrm{ML}$ is not single frequency but is a comb of optical tones, the SL can lock to the nearest tone. This approach has been successfully tested on one of our dual wavelength chips as detailed in [5]. Nevertheless, such a solution is not versatile and is quite heavy in terms of implementation since it requires an additional stable laser to generate the comb.

To overcome this limitation, we propose a new approach which consists in generating a frequency comb around each DFB line and optically re-inject this dual-comb into the PIC. This principle is illustrated in Figure 2. The frequency comb around each optical line is generated using a phase modulator. The resulting partially overlapping combs are then delayed and sent back to the PIC. Their free spectral range is adjusted so that mutual optical injection occurs for a given tone of each comb. This scheme combines several advantages. (i) First it leads to spectral narrowing for each DFB line thanks to optical self injection after a delay in the fiber loop. (ii) Second, the loop can ensure crossstabilization. Indeed, one comb line generated with $u$ can inject $v_{2}$ and, simultaneously, one comb line generated with $v$ can inject $u$. By properly choosing the modulation frequency $f_{R F}$, it is possible to match a given harmonic issued from the first laser with the frequency of the second laser and vice-versa. In this case, $v_{2}=v_{1}+N . \mathrm{f}_{\mathrm{RF}}$, where $N$ is the harmonic number of the injected tone.

Both DFBs are optically self-injected as well as mutually cross-injected. One could then expect a significant and robust stabilization of the beatnote between the two DFBs of the PIC and consequently a strong reduction of the $\mathrm{mmW}$ carrier phase noise.

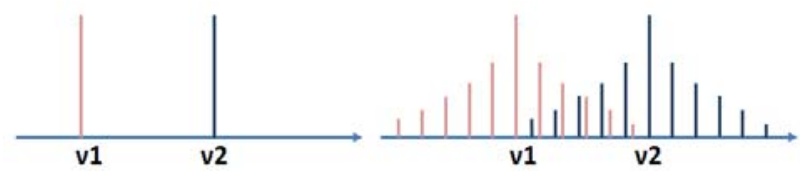

no modulation, no injection

modulation, no injection

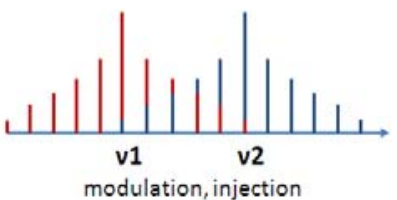

Fig.2. Optical spectrum: illustration of the cross self-injection phase locking.

The experimental setup is shown in Figure 3. Thanks to the optical output port of the PIC, the dual frequency signal is coupled in a lensed fiber and sent into an optical circulator. Following the light direction, a phase modulator (MФ) is used to generate the optical frequency comb around each DFB laser line. It is driven with a $33 \mathrm{dBm} R F$ signal provided by a synthesizer followed by an $R F$ amplifier. The phase modulator is a Photline MPZ-LN-10 presenting a $10 \mathrm{GHz}$ bandwidth and a low $V \pi$ of $5 \mathrm{~V}$ allowing efficient harmonics generation when driven with a proper RF power. Such property is useful for locking the two lasers in a large range of optical frequencies. Due to the weakness of the optical signal in this proof of concept experiment, an Erbium Doped Fiber Amplifier (EDFA) is included in the loop for optical amplification. Moreover, an optical filter with $0.7 \mathrm{~nm}$ bandwidth is introduced after the EDFA to filter out the amplified spontaneous emission ensuring a relatively clean optical reinjection. After a cycle, the signal is injected back into the PIC through the lensed fiber. Two polarization controllers are used: the first one to adjust the beam polarization at the entrance of the phase modulator and the second one to control the polarization matching of the PIC output and re-injected signal. The electrical beat note and the optical spectrum are simultaneously monitored using an electrical spectrum analyzer (ESA) and an optical spectral analyzer (OSA) respectively. 


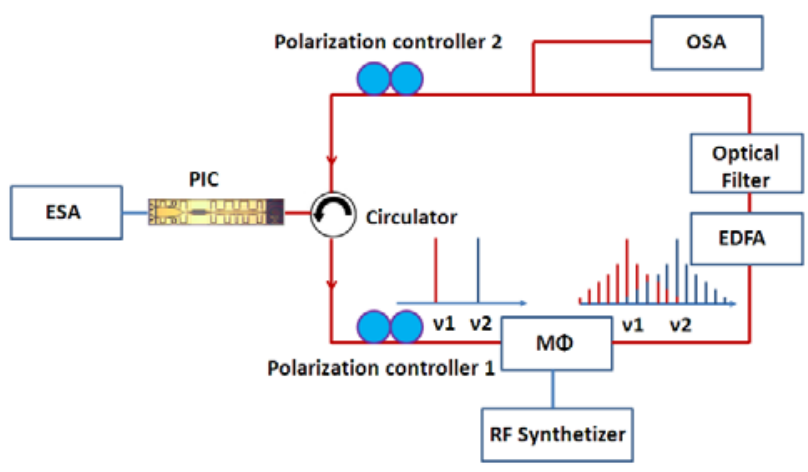

Fig.3. Schematic representation of the self injection setup

DFB1 and DFB2 are biased so that the beatnote frequency delivered by the PIC is $90 \mathrm{GHz}$ which corresponds to a wavelength difference of $0.72 \mathrm{~nm}$ between the two DFBs oscillating at around $1562 \mathrm{~nm}$. After been amplified on SOA sections 1.2 and 1.3, the signal is sent to the $-3 \mathrm{~V}$ biased photodiode UTC1 generating a photocurrent of $8.2 \mathrm{~mA}$. The optical power coupled in the circulator is estimated to be $15 \mu \mathrm{W}$. This low coupling efficiency is due to the high divergence of the output beam. The lasers present however a good single-mode behavior with SMSR higher than $33 \mathrm{~dB}$. Finally, the PIC temperature is maintained at $20^{\circ}$ with a thermoelectric cooler to improve the long-time stability of the system.

After passing through the phase modulator, harmonic tones are generated on both sides of each laser line. In Figure 4, we show a comparison of the optical spectra with and without modulation, both measured after the optical filter, i.e., just before reinjection. As can be noticed, these spectra are affected by the response of the optical filter.

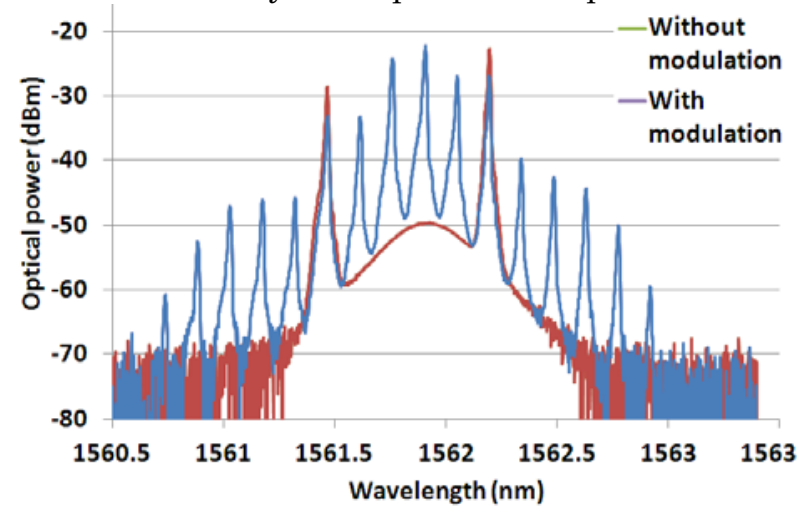

Fig.4. Optical spectrum with and without phase modulation, before reinjection.

We measure the corresponding generated electrical spectrum delivered by the monolithically integrated high speed photodiode. To this aim the signal is electrically down-converted with an external mixer prior analysis with an ESA. In Figure 5, we show how behaves the mmW tone when the optical feedback loop is closed but no signal is applied to the phase modulator.

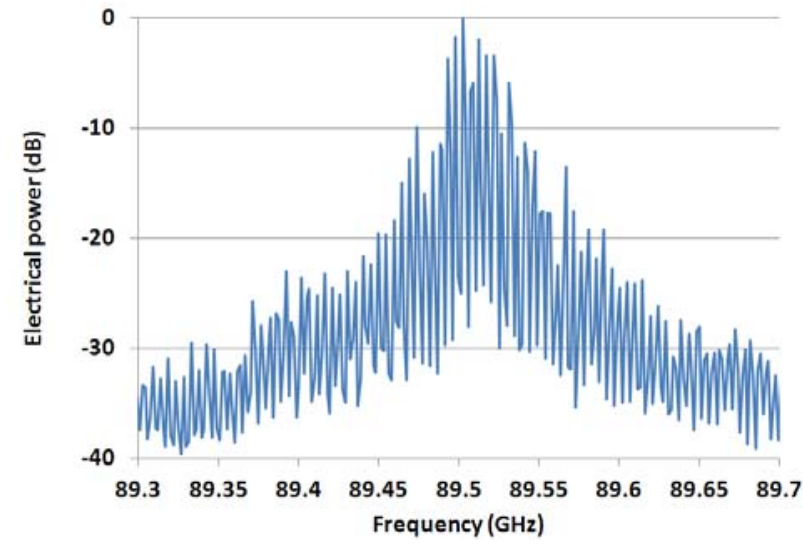

Fig.5. Electrical spectrum of the beatnote when the PIC is injected by the external fibered setup without RF modulation (RBW=200 $\mathrm{kHz}, \mathrm{Span}=400 \mathrm{MHz}$ ).

We can identify on this spectrum the filtering effect of the optical loop which acts in these conditions as a simple external cavity coupled to the two DFB lasers. This external cavity slices the wide $\mathrm{mmW}$ tone into discrete tones having a $5 \mathrm{MHz}$ free spectral range, which corresponds to a 40 meter optical path in agreement with the total length of the loop composed of the different fibered elements.

By finely adjusting the modulation frequency around 18 $\mathrm{GHz}$ on the phase modulator, one reaches periodically the situation where the fifth harmonic of the RF signal lies in the vicinity of the original free running beatnote $\left(v_{2}-v_{1}=5 . f_{\mathrm{RF}}\right)$ and where it matches one of the feedback loop longitudinal modes. By this way, a narrow linewidth beatnote strongly emerges as shown in Fig.7. and we observe a consequent reduction of the power of the non-injected modes, illustrating a nice example of energy transfer. Figure 6 shows the comparison between the electrical spectra of the original free running beatnote and the successfully phase locked beatnote. We can notice the strong emerging power of the selected mode, i.e., close to 15 $\mathrm{dB}$ increase as compared to the free running regime.

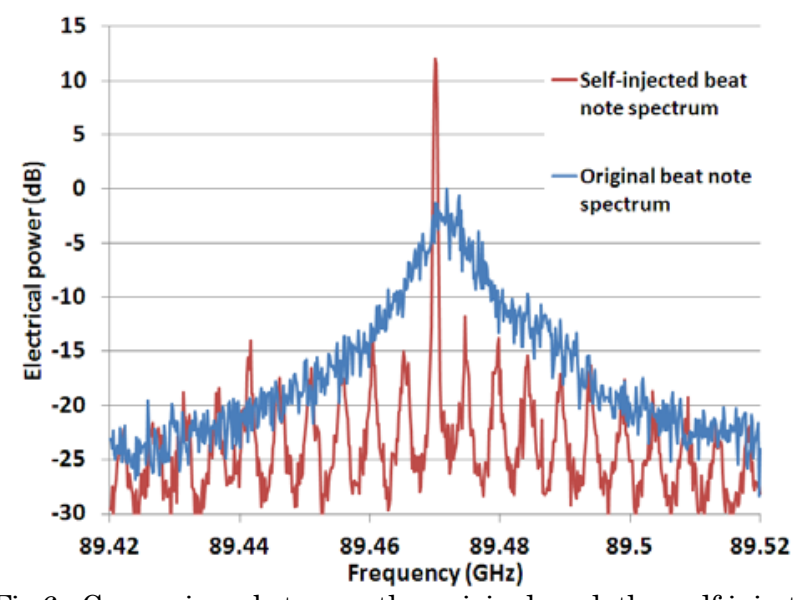

Fig.6. Comparison between the original and the self-injected beatnote spectrum (RBW=300 kHz, Span=100 MHz). 
These results clearly prove that two mechanisms are involved for the spectral narrowing: the feedback loop which creates in a first time a filter slice and, in a second time, the efficient optical cross-injection which select one of these tones and bring up this selection. It leads to the huge reduction of the beatnote linewidth and the concentration of energy on one mode. In the cross-injected operating mode, the beatnote linewidth at half maximum drops below $30 \mathrm{~Hz}$ (limited by the resolution of our ESA). This excellent result is in the same order of magnitude as dualfrequency solid-state lasers [12,13] but in a monolithically integrated semiconductor laser.

The corresponding phase noise power spectral density of the self-injected beatnote was measured experimentally, as shown in Fig. 7, using the phase noise setting of the ESA.

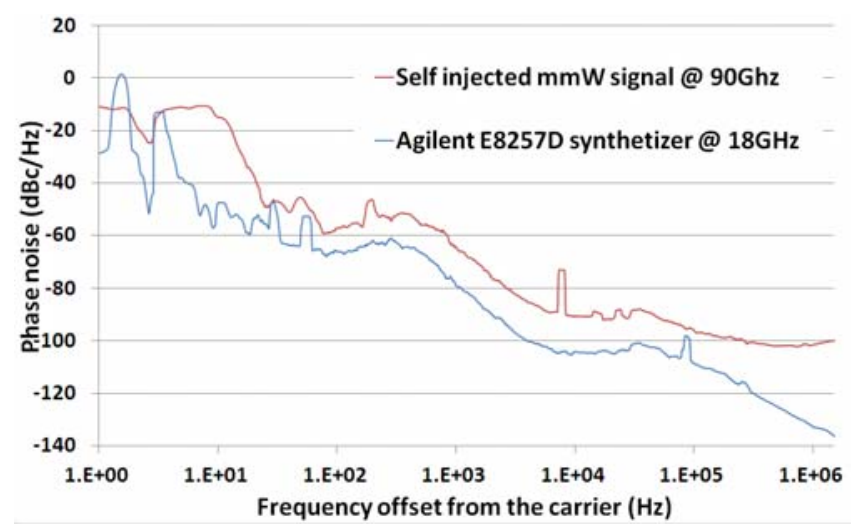

Fig.7. Comparison between the synthetizer and the selfinjected beatnote phase noise

The phase noise level of the self injected beatnote is below $-90 \mathrm{dBc} / \mathrm{Hz}$ for frequency offsets from the carrier of $10 \mathrm{kHz}$ and higher attesting the high purity of the signal. The phase noise spectral density of the synthesizer is shown for comparison. The additive noise is evaluated about to $14 \mathrm{~dB}$ for frequencies between $100 \mathrm{~Hz}$ and $10 \mathrm{kHz}$ corresponding to the theoretical expected degradation for the fifth harmonic of the synthesizer frequency following the equation (2):

$$
L\left(f_{n}\right)=L\left(f_{0}\right)+20 \log (n)
$$

With $L\left(f_{n}\right)$ the phase noise of the nth harmonic and $\mathrm{L}\left(\mathrm{f}_{0}\right)$ the phase noise of the original synthesizer note. Above $100 \mathrm{kHz}$, the phase noise reaches the optical shot noise floor.

In conclusion, the phase locking of a monolithically integrated photonic $\mathrm{mmW}$ source has been successfully demonstrated using a high order optoelectronic cross injection technique. The proposed technique consists in generating around each wavelength an optoelectronic frequency comb that is optically re-injected in our fully integrated photonic mmW source. The combination of self and mutual cross-injection lead to robust and high spectral purity. The linewidth of the beat note at $90 \mathrm{GHz}$ was reduced below $30 \mathrm{~Hz}$ and the phase noise level below -90 $\mathrm{dBc} / \mathrm{Hz}$ for frequency offsets from the carrier of $1 \mathrm{kHz}$ or more which is the state of the art for this kind of monolithically integrated device. The power spectral density of the $\mathrm{mmW}$ carrier being significantly improved, the next step will be to perform a transmission at high data rate with this setup. These results support the promising prospects of monolithically integrated photonic $\mathrm{mmW}$ sources in future communication networks.

This work was supported by the European Commission within FP7 ICT European project iPHOS and FP7 IPHOBAC-NG project.

\section{References.}

[1] Stöhr, A. IEEE International Topical Meeting on Microwave Photonics 2010

[2] Lu, M., Park, H., Bloch, E., Sivananthan, A., Parker, J., Johansson, L.A., Mark, Rodwell, M., Coldren, L., IEEE Photonics Technology Letters, 2013 [3] van Dijk, F., Kervella, G., Lamponi, M., Chtioui, M., Lelarge, F., Vinet, E., Robert, Y., Fice, M.J., Renaud, C.C., Jimenez, A., Carpintero, G., IEEE Photonics Technology Letters, (Volume:26, Issue: 10), 2013

[4] Gliese, U., Nielsen, T.N., Bruun, M., Lintz Christensen, Ë. Stubkjaer, K.E., Lindgren, S., Broberg, B., IEEE Photonics Technology Letters., 1992,4, (8), pp. 936-938

[5] Balakier, K., Fice, M., van Dijk, F., Kervella, G., Carpintero, G., Seeds, A., Renaud, C., Optics Express, Vol. 22, Issue 24, pp. 29404-29412 (2014)

[6] Kervevan, L., Gilles, H., Girard, S., Laroche, M., Optics Letters., 2007, 32, (9), pp. 1099-1101

[7] Wang, L., Romanelli, M., van Dijk, F., Vallet, M., ELECTRONICS LETTERS, 13th March 2014 Vol. 50 No. 6 pp. $451-452$

[8] Soldo, M., Gibbons, N., Giuliani, G., CLEO 2009

[9] van Dijk, F., Lamponi, M., Chtioui, M., Lelarge, F., Kervella, G., Rouvalis, E., Renaud, C., Fice, M., Carpintero, G., Microwave Photonics (MWP), 2013 International Topical Meeting on

[10] Kervella, G., Chtioui, M., Lamponi, M., Robert, Y. Vinet, E., Make, D, Fice, M. Renaud, C. Carpintero, G. van Dijk, F. 2014 IEEE Photonics Conference, IPC $2014226-227$

[11] Stover, H. L. and Steier, W. H., Appl. Phys. Lett. 8, 91 (1966)

[12] Pillet, G., Morvan, L., Menager, L., Garcia, A., Babiel, S., Stohr, A., Journal of Lightwave Technology, 32(20), 3824-3830. (2014).

[13] Rolland, A, Loas, G., Brunel, M., Frein, L., Vallet, M., Alouini, M., Opt. Exp., vol. 19, pp. 17944-17950, (2011). 